\title{
COMPETITIVIDAD SALARIAL DEL SECTOR INDUSTRIAL Y AGROINDUSTRIAL EN LA CIUDAD DE VILLAVICENCIO, COLOMBIA
}

\section{WAGE COMPETITIVENESS IN THE INDUSTRY AND AGROINDUSTRY SECTORS IN VILLAVICENCIO, COLOMBIA}

\author{
Dagoberto Torres Flórez*, Danae Duana Ávila** \\ Alexander Almeida Espinosa ${ }^{\star \star \star}$
}

* Doctorando en Ciencias Económicas y Administrativas. Universidad de los Llanos. Email: dtorres@unillanos.edu.co. ORCID: http://orcid.org/0000-0002-7925-3005.

** Doctor en Ciencias Económico Administrativas. Universidad Autónoma del Estado de Hidalgo. Email: duana@uaeh.edu.mx. ORCID: http://orcid.org/0000-0003-2286-2843.

*** PhD Ciencias de la Salud - Doctor en Ciencias Económicas y Administrativas. Universidad Industrial de Santander. Email: alexanderalmeida10@gmail.com. ORCID: http://orcid.org/0000-0002-5597-0405.

Dirección para recibir correspondencia: duana@uaeh.edu.mx 
COMPETITIVIDAD SALARIAL DEL SECTOR INDUSTRIAL Y AGROINDUSTRIAL EN LA CIUDAD DE VILLACENCIO, COLOMBIA.

\section{RESUMEN}

El estudio de competitividad salarial del sector industrial y agroindustrial de la ciudad de Villavicencio 2017.

OBJETIVO: Analizar el comportamiento de las empresas frente a aspectos de orden de remuneración laboral, incentivos, beneficios sociales y demás aspectos que intervienen en el desarrollo del sector industrial de la ciudad; esta información será útil para la coordinación y aplicación de políticas y programas orientados a impulsar la competitividad de estos sectores; como también un referente para las empresas que deseen incursionar en el mercado regional, para que estas conozcan las condiciones salariales en las que se encuentra actualmente los sectores económicos de la región.

MATERIAL Y MÉTODO: Estudio descriptivo y cuantitativo con el fin de conocer la percepción que tiene cada una de las empresas del sector industrial y de servicios de la ciudad de Villavicencio, con respecto al pago de salarios y compensaciones que cada una de ellas hace a sus colaboradores.

RESULTADOS. Los puestos que ocupa el personal requieren un nivel de educación profesional, por otro lado, el número de horas trabajadas es acorde al salario pagado en el sector agro industrial.

CONCLUSIONES: El salario que obtienen los colaboradores de las empresas del sector servicio son de acuerdo a su nivel de escolaridad y su nivel de experiencia, debido a que es importante que tengan el conocimiento y las habilidades.

PALABRAS CLAVE: Salario. Remuneración económica. Sector industrial. Sector agroindustrial. Competitividad salarial.

\section{ABSTRACT}

This is a study carried out on wage competitiveness in the industry and agroindustry ector in Villavicencio in 2017.

OBJETIVO: To analize companies behavior related to remuneration aspects, incentives, 
COMPETITIVIDAD SALARIAL DEL SECTOR INDUSTRIAL Y AGROINDUSTRIAL EN LA CIUDAD DE VILLACENCIO, COLOMBIA.

socialbenefits and other related aspects that intervene in the industry sector in the city; this information will become handy when coordinating or applying programs and policies oriented to the boost of competitiveness of both sectors; and also as a reference for companies that are trying to enter in the regional market, so they are aware of the current salary conditions.

MATERIAL AND METHOD: Quantitative descriptive study directed to retrieve companies perception on salary and compensations conditions of their colleagues in the industry and services sectors in Villavicencio.

RESULTS: Professional education is required in order for personnel to be in charge of a position, and also, the payment is calculated according the man hours worked in the agroindustry sector.

CONCLUSIONS: The salary obtained by colleagues in the industry sector is according to their level of education and experience, considering that knowledge and skills are required for the positions.

KEY WORDS: Wage. Economic Remuneration. Industry Sector. Agroindustry Sector. Wage Competitiveness

\section{INTRODUCCIÓN}

En la actualidad, el Gobierno Nacional de Colombia y las empresas afrontan decisiones que impactan de forma determinada el comportamiento del sector productivo colombiano y el de la sociedad en general; esto se observa, debido a que el gobierno impone leyes sobre diferentes aspectos que influyen en el valor de la mano de obra calificada y no calificada, de cada uno de los sectores productivos del aspecto económico del país.

Asimismo, las condiciones de la economía nacional, brindan oportunidades y el alcance de expectativas laborales que pueden ser interpretadas por cada uno de los actores del sistema de forma distinta; pero son las empresas las que "crean bienes y servicios con valor agregado usando métodos eficientes. Solamente de esta forma una nación puede mantener altos salarios y atractivos retornos de capital para apoyar una inversión sostenida" (Lombana \& Silvia, 2008), en este contexto la productividad de la empresa se basa en dos pilares interrelacionados: la 
COMPETITIVIDAD SALARIAL DEL SECTOR INDUSTRIAL Y AGROINDUSTRIAL EN LA CIUDAD DE VILLACENCIO, COLOMBIA.

sofisticación de la empresa para operar en el país donde compite y la calidad del ambiente microeconómico en el que la empresa compite.

La productividad laboral en Colombia, de acuerdo con el indicador construido por el Conference Board, señala un informe de Fedesarrollo, ocupa uno de los peores lugares entre los países de la región, lo cual posiciona al país en el quinto lugar de América Latina, dato que se complementa con el reportado por el Foro Económico Mundial, entidad que lo ubicó en el puesto 61 entre 140 países evaluados del Índice Global de Competitividad (ICG). (Portafolio, 2016). Esto representa un panorama desalentador para la microeconomía del país, puesto que la baja productividad en el trabajo termina desencadenando inadecuados resultados de formalización y salarios, provocando que estos empeoren y trasciendan de forma negativa en el periodo de contratación. Esto hace que el bienestar de la población se vea directamente afectado, e implica la disminución de los niveles de calidad de vida y de confianza en los distintos mercados locales.

El ICG encontró que Colombia ha caído en el ranking principalmente, por malos desempeños en eficiencia del mercado laboral y preparación tecnológica, ambos con una regresión notable entre 2010 y 2015. Específicamente, el punto del mercado laboral muestra que los empleadores locales han generado este estancamiento, lo cual se ve reflejado en que el país bajó del puesto 69 en 2010 al 86 en 2015, debido a las malas prácticas de contratación y despido registradas en el periodo de tiempo. Esto dispone un escenario en donde la economía colombiana, además de estar caracterizada por una informalidad muy elevada, está dada por un uso inapropiado y poco eficiente de los recursos laborales, lo que a su vez repercute de forma negativa a los colombianos asalariados de empresas pequeñas en el país.

La Federación Colombiana de Gestión Humana (ACRIP) realizó un estudio que permite identificar la estructura salarial en la actualidad mediante un mapa del mercado laboral colombiano y allí explica cuáles son los sectores económicos mejor y peor remunerados en el país en el 2017 (Colombia, 2017) analizando más de 900 cargos de 1.055 empresas colombianas y multinacionales, distribuidas en 14 de las ciudades más importantes del país y relacionadas con los 24 sectores de la economía de la nación.

El resultado del estudio muestra que los empleados públicos tuvieron un incremento salarial promedio de 6,75\% durante este año, para un 59\% por encima del promedio nacional, mientras que el de minería, energía e hidrocarburos un $44 \%$, el químico fármaco $43 \%$ y el de servicios financieros y de seguros $25 \%$. Caso contrario es el de hotelería y turismo, que remunera un $45 \%$ 
COMPETITIVIDAD SALARIAL DEL SECTOR INDUSTRIAL Y AGROINDUSTRIAL EN LA CIUDAD DE VILLACENCIO, COLOMBIA.

por debajo, siendo el de peor proyección en el ranking (Trabajo, 2017).

\section{ESTRUCTURA SALARIAL Y POLÍTICAS SALARIALES}

Dentro de una organización, son el eje fundamental para tener una fiscalización concreta sobre los recursos económicos disponibles, con la que cuentan las organizaciones; de igual forma, los trabajadores están en la obligación de reconocer de donde proviene su remuneración salarial y sueldo. De no contar con la claridad de la información de este recurso, puede generarse situaciones de inconformidad entre los trabajadores y habrá desigualdades entre los perfiles e ingresos para cada cargo.

La alta preocupación de la alcaldía de Villavicencio por el alarmante índice de desempleo en la ciudad, prendió alertas y estrategias efectivas de seguimiento y verificación, sumado al aporte de acciones inmediatas que favorezcan la empleabilidad en la ciudad, debido a que son más las personas que no cuentan con un contrato legal de trabajo formal; por lo tanto, la economía informal sigue persistiendo actualmente en la ciudad; para las autoridades, el desempleo es uno de los factores más agobiantes en la región, se estipula que más de 43,000 personas están sin empleo correspondiente a todo el trimestre del 2016, las estadísticas del DANE revelan que la ciudad cuenta con una tasa de desocupación del 13.0\%, considerada como la séptima ciudad del país con esta grave estadística (Redacción , 2009).

\section{SISTEMA SALARIAL O DE COMPENSACIONES}

Para Morales y Velandia (1999) es el conjunto de políticas, técnicas y objetivos de compensación traducidos en las retribuciones financieras, prestaciones o beneficios tangibles que se dan a los empleados como parte de la relación laboral. Sin embargo, (Chiavenato, 2012) define el sistema de compensaciones como la remuneración producto del salario directo más el salario indirecto, que representa todo lo que el empleado recibe como fruto del trabajo que realiza en la organización.

El concepto de compensación incluye todo tipo de recompensas, tanto extrínsecas como intrínsecas, que consideran elementos tanto monetarios como no monetarios, que son recibidas por el empleado como resultado de su trabajo en la organización. Las recompensas extrínsecas se refieren al sueldo base o salario, incentivos o bonos y beneficios distribuidos directamente por la organización. Las recompensas intrínsecas se refieren a recompensas internas del individuo, derivadas de su involucramiento en ciertas actividades o tareas, como son: satisfacción laboral, 
COMPETITIVIDAD SALARIAL DEL SECTOR INDUSTRIAL Y AGROINDUSTRIAL EN LA CIUDAD DE VILLACENCIO, COLOMBIA.

compromiso, autonomía, oportunidades de crecimiento y aprendizaje (Villanueva y Gonzáles, 2005).

\section{SALARIOS}

La fuerza de trabajo como factor de producción se compra y se vende en el mercado laboral: los trabajadores ofrecen su fuerza y su tiempo para realizar dicha actividad dentro de una empresa tanto pública como privada, donde el empleador ofrece una contraprestación, es decir, un salario por la realización del tiempo laborado en su cargo, lo cual se convierte en una manera de incentivar al empleado, para que cada día sea más competitivo y demuestra mejores resultados dentro de la organización.

Los salarios se deben asignar teniendo en cuenta varios criterios como la valoración del cargo, valoración del desempeño, valoración de utilidades del periodo, valoración de los salarios del mercado basándose en la capacidad de la empresa, en su competencia, en los requisitos del cargo y el desempeño extraordinario del personal (González, 2006).

Algunas teorías sobre la determinación del salario y sus implicaciones económicas en diversos contextos (ver tabla 1).

\section{Tabla 1}

Teoría de los salarios

\begin{tabular}{|c|c|}
\hline Autor & Teoría \\
\hline $\begin{array}{l}\text { Smith (1723-1790): } \\
\text { Teoría de los ingresos }\end{array}$ & $\begin{array}{l}\text { Tanto la demanda como la oferta influyen en la fijación de los salarios ya que el } \\
\text { salario se debe establecer comparando los que se ofrezcan en el mercado con } \\
\text { lo que ofrecen las partes empresarias, para así llegar a un acuerdo en los } \\
\text { aumentos del salario mínimo. "el salario debe ser el necesario para que el } \\
\text { trabajador y su familia subsistan a lo que denomina el nivel de subsistencia, la } \\
\text { demanda de obra asalariada aumenta con el incremento del ingreso y del capital } \\
\text { de naciones" (Smith, 1723). }\end{array}$ \\
\hline $\begin{array}{l}\text { David Ricardo } \\
\text { La teoría de la subsistencia }\end{array}$ & $\begin{array}{l}\text { David Ricardo supone que, si el salario de mercado se eleva por encima del } \\
\text { nivel natural, aumentará el número de población, es decir las familias se } \\
\text { reproducirán en mayor proporción, lo que aumentaría la oferta de trabajo y } \\
\text { disminuiría el salario de mercado (Ricardo, 1900). }\end{array}$ \\
\hline $\begin{array}{l}\text { Karl Marx } \\
\text { Teoría de los salarios }\end{array}$ & $\begin{array}{l}\text { Con muy poca frecuencia el trabajador recibe unos ingresos superiores al nivel } \\
\text { de subsistencia, en su obra El Capital "El valor de la fuerza de trabajo está } \\
\text { determinado por el valor de los medios de subsistencia que habitualmente } \\
\text { necesita el obrero medio, tal valor, como el de cualquier otra mercancía, se } \\
\text { determina por el tiempo de trabajo necesario para su producción" (Marx, 1867). }\end{array}$ \\
\hline $\begin{array}{l}\text { Los neoclásicos refrescan las ideas } \\
\text { clásicas y postulan }\end{array}$ & $\begin{array}{l}\text { Los salarios son una suerte de precio que se determina por oferta } \\
\text { (trabajadores) y demanda (empresas). Esta teoría se funda principalmente en } \\
\text { la productividad marginal y tiene como fundamentos principales: una teoría de } \\
\text { la empresa y el supuesto que los mercados de productos y de trabajo son } \\
\text { perfectamente competitivos (Mujika, 1998). }\end{array}$ \\
\hline
\end{tabular}

TORRES-FLÓREZ D., DUANA-ÁVILA D., ALMEIDA-ESPINOSA A. 


\section{COMPETITIVIDAD SALARIAL DEL SECTOR INDUSTRIAL Y AGROINDUSTRIAL EN LA CIUDAD DE VILLACENCIO, COLOMBIA.}

Teoría de los

Salarios de eficiencia

John Stuart Mill

La Teoría de fondo de los Salarios

La teoría del salario de equilibrio

J. Stacey (Adams)

Teoría de la equidad propuesta

Víctor Vroom

La teoría de la expectativa

Teoría de la motivación

Frederick Herzberg

La Teoría de los dos factores

Edward Locke 1968

La Teoría de las metas

Henry Ford

Teoría de los salarios
Los salarios son el reflejo de la productividad de los trabajadores. Sin embargo, funciones de producción como la de Solow (1979) definen a la productividad en función del salario real (Caraballo, 1996).

La oferta y demanda de trabajo eran fundamentalmente las que definen los salarios. Para John Stuart Mill, los salarios dependen de los ingresos de los dueños del capital por motivos de producción y el nivel salarial se dispone de acuerdo con lo que los empresarios están dispuestos a pagar para contratar la mano de obra (Stuart Mill, 1848).

En esta teoría, la clase obrera se ve en desventaja ya que no hay salarios mínimos ni protección social, su sueldo radica únicamente en el número de trabajadores disponibles para el mercado (acogida en el liberalismo económico). (Keynes, 2000).

Plantea que toda persona compara sus atributos y contribuciones a la organización con las demás personas que hacen parte de esta misma, con el fin de evaluar si la contribución por ese esfuerzo compensa este (Adams, 2007).

Expone que la motivación es el resultado de multiplicar tres variables: El deseo que posee cada persona por alcanzar su objetivo propuesto, la expectativa acerca de lo que traerá como resultado su labor y la recompensa que trae consigo esa labor (Vroom, 2012).

Prácticamente todo el comportamiento de los seres humanos es motivado. Las necesidades e impulsos crean estados de tensión interna que funcionan como input para que el organismo analice su entorno y procure satisfacer dicha necesidad. Esta dinámica puede ser comprendida a la luz del Modelo de Davis y Newstrom, en él, la motivación ocurre como consecuencia de una necesidad que provoca tensión en el organismo (Newstrom \& Davis, 2010).

Exponía que existen dos dimensiones a lo que se refiere la satisfacción laboral: la motivación, y la higiene. Según él, las necesidades de higiene son influenciadas por las condiciones físicas y ambientales del trabajo. La satisfacción de los empleados se alcanzaría mediante factores de higiene entre los cuales se pueden mencionar la supervisión, las relaciones interpersonales, las condiciones físicas de trabajo, el salario, entre otras (Herzberg, 2013).

Luchar por alcanzar una meta es vista como la mayor motivación en el trabajo y produce un nivel más alto de resultados. El ser humano actúa para satisfacer sus necesidades, entonces, los gerentes deben encontrar la forma de adaptar estas necesidades con las condiciones de la empresa mediante opciones motivantes que den la posibilidad de lograr esta satisfacción (Locke, 1968).

Establece que brindar salarios altos a los trabajadores conduce a que haya un mayor consumo, un incremento en la demanda de productos, y como consecuencia de esto, productos a precios bajos. Si la productividad crece, aumentan las utilidades y da lugar a nuevas inversiones que impulsan una mejor producción (Ford, 2013).

Fuente: Elaboración propia, adaptada de las teorías salariales propuestas por cada autor. 
COMPETITIVIDAD SALARIAL DEL SECTOR INDUSTRIAL Y AGROINDUSTRIAL EN LA CIUDAD DE VILLACENCIO, COLOMBIA.

\section{INCENTIVOS}

En toda organización se maneja un sistema de compensaciones, que busca incrementar la motivación de los colaboradores y su productividad. En mercado del siglo XXI, el trabajo día tras día es cada vez más globalizado, un mercado marcado por empresas que buscan remunerar la labor de sus colaboradores de forma equilibrada, ofreciendo compensaciones salariales similares y cercanas a un pleno empleo. Los incentivos más importantes o comunes son el dinero, el reconocimiento social, la alabanza, el aplauso. Un incentivo es un incitador a la acción, es un motivo visto desde fuera, es lo que vale para un sujeto, es lo que le atrae.

Para Guihard (2016) relaciona a los programas de incentivos laborales con estimular el rendimiento de los colaboradores mediante el uso de recompensas dirigidas a metas específicas, indica de igual forma que estos programas están ligados a los colaboradores del área de ventas de las empresas para alcanzar objetivos cuantitativos y generar compromiso en la fuerza laboral. Sin embargo, también alude al hecho de que es necesario estipular y comunicar correctamente los lineamientos para la entrega de la bonificación o incentivo.

Los incentivos a su vez, se clasifican de tal modo que los económicos se miden, por lo general, en términos monetarios.

\section{BENEFICIOS SOCIALES}

Los beneficios son comúnmente entendidos como el componente no monetario de la compensación total, como son: las vacaciones, los seguros de vida y salud, los convenios, plan de retiro, entre otros. Estos elementos dependen, principalmente, del tipo de organización, el tipo de cargo y el nivel jerárquico (Villanueva \& González, 2005). Los mercados laborales actuales, altamente competitivos, obligan a los responsables de recursos humanos de las empresas a desarrollar e implementar herramientas que colaboren con la motivación y la retención de sus colaboradores calificados.

Se denominan beneficios sociales a las prestaciones de naturaleza jurídica de seguridad social, no remunerativa en dinero, que brinda el empleador al colaborador y que tiene como objeto mejorar la calidad de vida del dependiente o de su familia a cargo. Chiavenato (1993) afirma que los beneficios sociales forman parte de la remuneración total y se dirigen a todos los miembros de la organización, sin tener en cuenta el puesto que desempeñen. Se define como medios indispensables de complemento y apoyo, proporcionado y financiado por la empresa, para 
COMPETITIVIDAD SALARIAL DEL SECTOR INDUSTRIAL Y AGROINDUSTRIAL EN LA CIUDAD DE VILLACENCIO, COLOMBIA.

estimular y mantener la fuerza de trabajo en un nivel satisfactorio de moral y productividad.

\section{COMPETITIVIDAD}

El modelo de la ventaja competitiva, se lleva a cabo a través de estrategias competitivas, que permitan tomar acciones ofensivas para sobreponerse a otras empresas del mismo rubro y obtener una posición beneficiosa y defendible en una industria. Asimismo, tiene como finalidad ayudar a la empresa a hacer frente a la competencia y lograr tener éxito, lo, que se traducirá en beneficios y ganancias económicas. (Porter, 1991) denomina la ventaja competitiva como el valor diferencial que una empresa crea para sus clientes, bien sea en forma de precios menores al de la competencia o por la diferenciación de productos; es decir, cualquier característica creada por una empresa para distinguirse del resto y la sitúa en una posición superior para competir.

La competitividad es un concepto que no tiene límites precisos y se define en relación con otros conceptos. La definición operativa de competitividad depende del punto de referencia del análisis -nación, sector, firma-, del tipo de producto analizado -bienes básicos, productos diferenciados, cadenas productivas, etapas de producción- y del objetivo de la indagación -corto o largo plazo, explotación de mercados, reconversión (Pineiro, 1993).

\section{MATERIAL Y MÉTODO}

La investigación se realizó con un enfoque cuantitativo basado en el estudio y análisis de la realidad a través de procedimientos que se basaron en la medición en apoyo a instrumentos de consulta y análisis, tales como: cuestionarios, con el objeto de interpretar y establecer un análisis de la situación. Lo anterior, corresponde a un tipo de estudio descriptivo, con el fin de conocer la percepción que tiene cada una de las empresas del sector industrial y de servicios de la ciudad de Villavicencio, con respecto al pago de salarios y compensaciones que cada una de ellas hace a sus colaboradores. Asimismo, la población en el sector industrial y agroindustrial en empresas pequeñas, medianas y grandes en la ciudad de Villavicencio, dicha información fue tomada de la base de datos suministrada por la alcaldía.

Se utilizaron dos cuestionarios; uno general MP05a compensaciones y otro más específico MP05b compensaciones por cargo. El modelo de medición de procesos de gestión humana en PYMES (Torres, 2016), menciona en su fase 1, su carácter descriptivo para desarrollar los objetivos propuestos de identificar las condiciones de contratación de personal, conocimiento de los aspectos salariales, describir los aumentos salariales e identificar los incentivos laborales. Se 
COMPETITIVIDAD SALARIAL DEL SECTOR INDUSTRIAL Y AGROINDUSTRIAL EN LA CIUDAD DE VILLACENCIO, COLOMBIA.

realizó aplicación de cuestionarios para determinar estos aspectos en las empresas del sector industrial y de servicios de la ciudad de Villavicencio, teniendo en cuenta la función de los aspectos salariales y sus impactos en las organizaciones establecidas en el marco teórico.

Se analizaron 40 empresas y 130 cargos del sector industrial y agroindustrial. A la hora de desarrollar el trabajo, en forma que los datos fueran claros y concisos para todo público que desee leer este proyecto y familiarizarse con los resultados y el análisis propuesto, se tuvieron en cuenta unos rangos, cada uno con su debida calificación para una mejor interpretación (ver tabla 2).

Tabla 2

Interpretación de los datos

\begin{tabular}{lcc}
\hline Rangos & Calificación & Interpretación de los datos \\
\hline $0-20 \%$ & Muy mal & Casi nunca \\
$21-40 \%$ & Mal & Muy pocas veces \\
$41-60 \%$ & Regular & Ocasionalmente \\
$61-80 \%$ & Bueno & Con frecuencia \\
$81-100 \%$ & Muy bueno & Casi siempre \\
\hline
\end{tabular}

Fuente: Elaboración propia.

En cuanto al análisis de los salarios se utilizaron los cuartiles (Q), que son medidas estadísticas de posición que tienen la propiedad de dividir la serie estadística en cuatro grupos de números iguales de términos (Q1), (Q2), (Q3), (Q4), en el trabajo se manejaron 3 cuartiles. El primer cuartil es el nivel de remuneración tal que es superado por el 75\% de la muestra, quedando el 25\% por debajo de este valor; el segundo cuartil es justo la mitad de las observaciones que están por debajo y la mitad que están por encima; el cuartil tres es el nivel de remuneración que es superado por el $25 \%$ de la muestra, quedando el $75 \%$ por debajo de este valor.

\section{RESULTADOS}

Los sectores industrial y agroindustrial, juegan un papel muy importante en la economía de la ciudad, por esto se decidió realizar un estudio de la competitividad salarial para estos dos sectores; teniendo en cuenta que el sector servicio es un sector demasiado grande, se excluyeron subsectores como el hotelero, turístico y de transporte, y se trabajaron en otros proyectos por aparte, pero con la misma finalidad propuesta por este proyecto. 
COMPETITIVIDAD SALARIAL DEL SECTOR INDUSTRIAL Y AGROINDUSTRIAL EN LA CIUDAD DE VILLACENCIO, COLOMBIA.

El sector industrial como el agroindustrial, aunque tienen una amplia participación en la base económica de la ciudad de Villavicencio, se viene notando con mayor intensidad su actividad y se espera a futuro que hagan un gran aporte tanto a la economía de la ciudad, así como también tengan una oportunidad de empleo a propios y visitantes.

\section{CARACTERÍSTICAS}

Los sectores industrial y agroindustrial el $78 \%$ de los cargos son cargos de carácter auxiliar, dicho cargo está representado en 50,09\% por personal técnico, 22,55\% de personal empírico, el $12,75 \%$ personal con nivel de educación bachiller, 7,84\% tecnólogos y en pequeñas proporciones personal con nivel primaria y profesional en un 3,92\% y 1,96\% respectivamente. El 11\% está representado por personal de cargo profesional y del mismo modo este $100 \%$ son colaboradores con nivel de educación profesional; Los asistentes y jefes de primera línea cada uno representan un $2 \%$ total de personal en dicha categoría de cargo, y en donde ser técnico es importante como requisito para ser asistente por otro lado para ser jefe de primera línea es requisito para unas organizaciones ser profesional en algunas otras tan solo saber o tener la disposición de aprender; es decir, ser empírico. Los administradores y los supervisores representan en la industria y agroindustria cada uno el 3\% del total del personal en donde no es requisito tener algún nivel de educación básica o superior para dicho cargo de administrador, mientras que para el cargo de supervisor puede ir desde empírico, técnico, tecnólogo e incluso profesional en algunas organizaciones (ver figura 1).

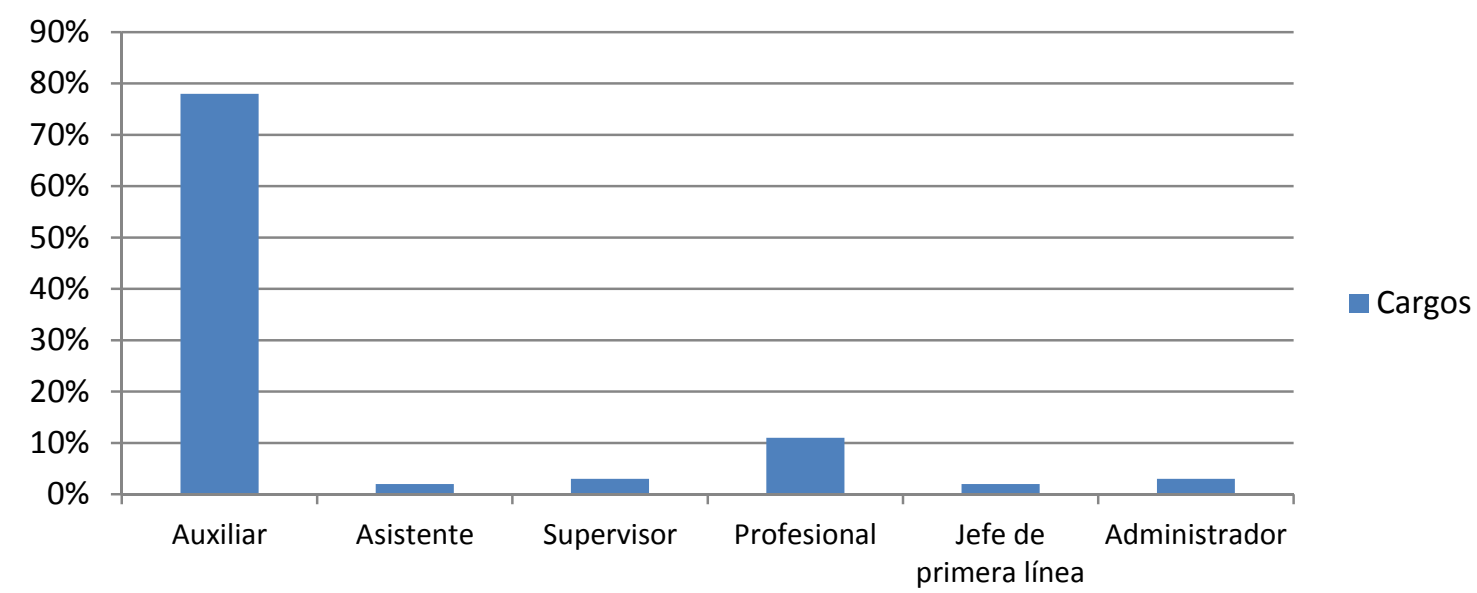

Figura 1. Promedio de los cargos más representativos en las empresas del sector industrial y agroindustrial en Villavicencio.

Fuente: Elaboración propia. 
COMPETITIVIDAD SALARIAL DEL SECTOR INDUSTRIAL Y AGROINDUSTRIAL EN LA CIUDAD DE VILLACENCIO, COLOMBIA.

El 80\% de las empresas del sector industrial y agroindustrial están representadas por empresas pequeñas. Para todos los efectos, son empresas pequeñas aquellas que el número de colabores redondea entre 11 y 50 colaboradores (Industria y turismo, 2012), por otro lado el 18\% de las empresas están representadas por medianas empresas ya que su equipo de colaboradores son mayores a 50 e iguales o menores a 200 según lo estipulado por la ley colombiana y finalmente la microempresa que representa el 3\% de las empresas en el municipio de Villavicencio, Meta puesto que el número de sus colaboradores es menos o igual a 10 (ver figura 2).

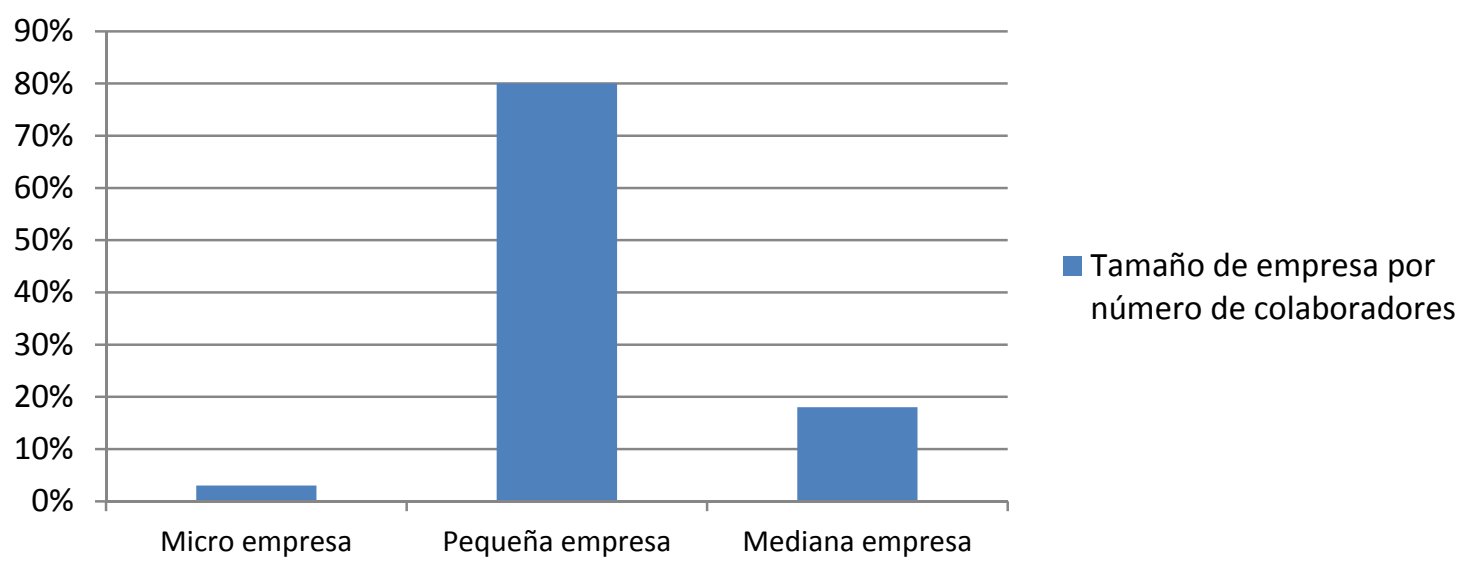

Figura 2. Clasificación del tamaño de las empresas del sector industrial y agroindustrial en la ciudad de Villavicencio.

Fuente: Elaboración propia.

Para Godoy (2001) la percepción de los empresarios en torno al desempeño laboral de mujeres y hombres y en especial a las supuestas diferencias de productividad y costos a ellos asociados, son factores que inciden en gran medida en las posibilidades de acceso de trabajadores de uno y otro sexo al empleo. En lo que refiere al sector industrial, es evidente que la inclusión laboral de la mujer es notablemente excluida por la naturaleza de la labor dentro del sector, en donde en gran mayoría las labores requieren de riesgo y fuerza bruta que aunque la mujer puede asumir; para el empresario es mucho más fácil y económico optar por la fuerza masculina. 
COMPETITIVIDAD SALARIAL DEL SECTOR INDUSTRIAL Y AGROINDUSTRIAL EN LA CIUDAD DE VILLACENCIO, COLOMBIA.

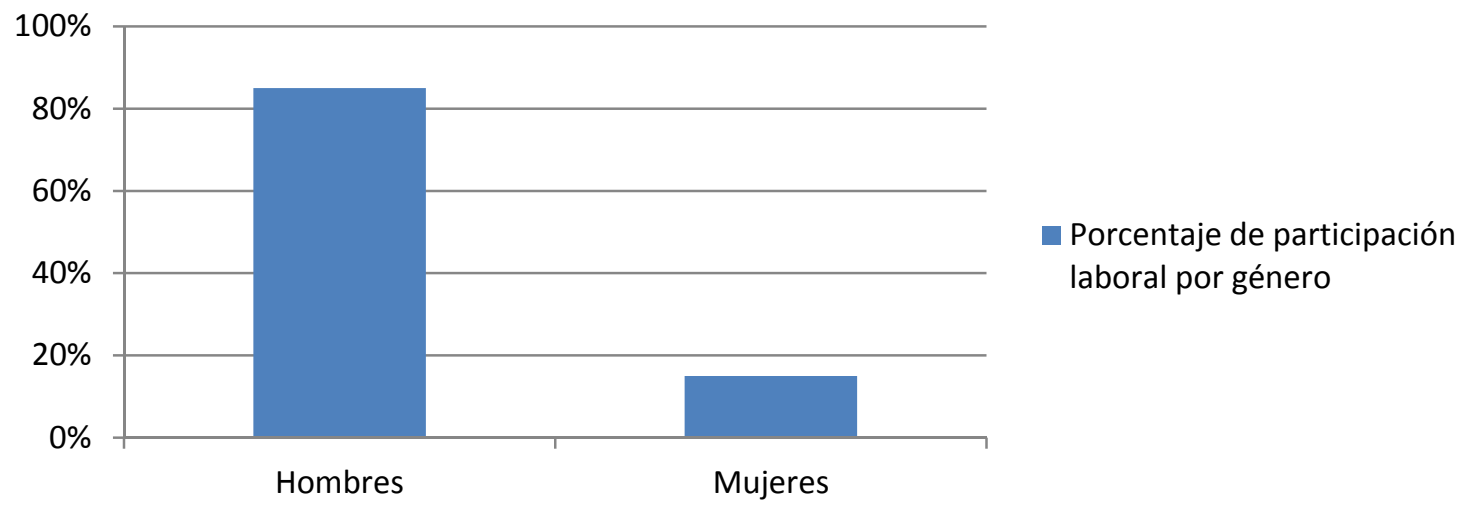

Figura 3. Promedio de colaboradores en las empresas del sector industrial y agroindustrial. Fuente: Elaboración propia.

El 85\% del personal que labora dentro de las organizaciones industriales y agroindustriales son representadas por hombres mientras que por otro lado el 15\% por mujeres lo que muestra una alta participación del personal masculino; dado caso se evidencia en consecuencia de las labores pesadas que se dan dentro de dichas organizaciones y el desgaste tanto físico como mental que requieren dichas actividades haciendo que el género femenino sea una alternativa para labores menos pesadas y más administrativas (ver figura 3).

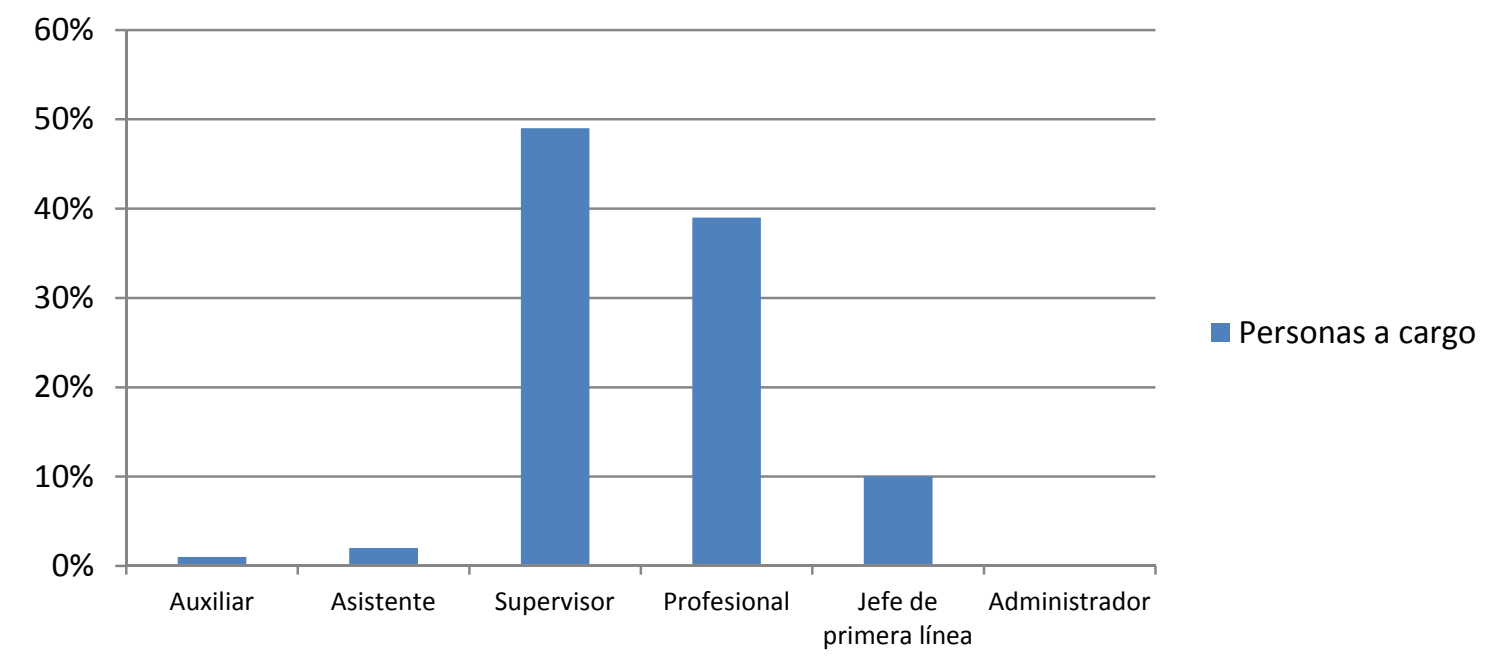

Figura 4. Promedio de cargos que manejan personal en el sector industrial y agroindustrial. Fuente: Elaboración propia.

Dentro de las organizaciones, el supervisor es uno de los cargos con mayor responsabilidad y dentro de las organizaciones industriales y agroindustriales no son la excepción, ya que es uno de los tres con mayor responsabilidad; ya que en promedio tiene a cargo entre 8 a 9 colaboradores, equivalentes a $49 \%$ del total de personal de dicha categoría en el sector, un profesional de 6 a 
COMPETITIVIDAD SALARIAL DEL SECTOR INDUSTRIAL Y AGROINDUSTRIAL EN LA CIUDAD DE VILLACENCIO, COLOMBIA.

7 colaboradores, representando el $39 \%$ de los colaboradores del sector y un jefe de primera línea manejan el $10 \%$ de los colaboradores, representados entre 1 y 2 personas, por otro lado el cargo de asistente auxiliar, podrían hacerse cargo de en promedio 1 persona, mientras que el cargos de administrador representa un cargo que no requieren tener bajo responsabilidad a personas, aunque represente un hecho atípico, puesto que los cargos de gerentes y administradores suelen ser los de mayor responsabilidad; en dicho caso los administradores resultan ser cuidadores de predios y no profesionales en administración de empresas (ver figura 4).

\section{Tabla 3}

Cargos más representativos según la categoría del cargo en el sector industrial y agroindustrial de la ciudad de Villavicencio

\begin{tabular}{|c|c|c|c|c|c|}
\hline Auxiliar & Asistente & Supervisor & Profesional & $\begin{array}{c}\text { Jefe de } \\
\text { primera línea }\end{array}$ & Administrador \\
\hline Conductor & Operario & Jefe de Bodega & Contador & Auxiliar & $\begin{array}{c}\text { Administrador } \\
\text { de Finca }\end{array}$ \\
\hline Secretaria & $\begin{array}{c}\text { Asistente } \\
\text { administrativo }\end{array}$ & & Zootecnista & Hornero & \\
\hline $\begin{array}{l}\text { Auxiliar de } \\
\text { bodega }\end{array}$ & & & & Director de Área & \\
\hline $\begin{array}{l}\text { Auxiliar } \\
\text { contable }\end{array}$ & & & & & \\
\hline
\end{tabular}

Fuente: Elaboración propia.

En la categoría auxiliar del sector industrial y agroindustrial, el $23 \%$ de los cargos de dicha categoría son cargos que desempeñan los conductores, el 17\% las secretarias, el 15\% los auxiliares de bodega, el 13\% los operarios y el $8 \%$ los auxiliares contables como cargos más representativos; los operarios dentro del sector pueden aspirar a ser auxiliares, asistentes y jefes de primera línea. El 71,49\% de los profesionales son contadores, el 14\% son profesionales HSQ y veterinarios y zootecnistas representan el 7\% cada uno; el 100\% de los administradores son administradores de fincas sin ningún tipo de formación educativa (ver tabla 3). 
COMPETITIVIDAD SALARIAL DEL SECTOR INDUSTRIAL Y AGROINDUSTRIAL EN LA CIUDAD DE VILLACENCIO, COLOMBIA.

Relación entre la educación, la experiencia y los salarios del sector industrial y agroindustrial en la ciudad de Villavicencio

La educación suele ser un factor que dentro de las organizaciones influye en gran parte, puesto que de ello depende el desarrollo del colaborador con respecto al desempeño de las actividades que exija directamente el puesto. Las carreras técnicas son elegidas cada vez con más frecuencia. Entre otras razones, se encuentran la alta demanda y la rapidez de inserción laboral; la fuerza que ha tomada este tipo de formación educativa, recae en la capacidad de solucionar problemas de manera práctica y rápida (Universia Chile, 2016).

Los colaboradores que se encuentran dentro de las organizaciones dedicadas a las labores industriales y agroindustriales y cuya categoría de cargo es auxiliar, asistente y jefe de primera línea; son personal en donde su cargo u/o labor en promedio requiere un nivel de educación bachiller, para el cargo de supervisor en promedio es requisito que tengan en un nivel de educación técnica y para un profesional que sea un nivel de educación profesional; mientras que por otro lado el hecho de aunque no se tiene un título pero sí; el conocer de una labor o el simple hecho de darse a la disposición de aprender de forma empírica representa mínimo de requisito para ser administrador (ver tabla 4).

Tabla 4

Promedio del nivel de educación en el sector industrial y agroindustrial en la ciudad de Villavicencio

\begin{tabular}{lc}
\hline Nivel de educación & Porcentaje \\
\hline Empírico & $23 \%$ \\
Primaria & $3 \%$ \\
Bachiller & $10 \%$ \\
Técnico & $42 \%$ \\
Tecnológico & $8 \%$ \\
Profesional & $14 \%$ \\
\hline
\end{tabular}

Fuente: Elaboración propia.

Por otro lado, la experiencia muchas veces resulta ser una barrera para acceder a un empleo y de acuerdo a la categoría del cargo y las diferentes políticas de las organizaciones varían las exigencias de meses o años; las organizaciones del sector industrial no son la excepción. Dentro 
COMPETITIVIDAD SALARIAL DEL SECTOR INDUSTRIAL Y AGROINDUSTRIAL EN LA CIUDAD DE VILLACENCIO, COLOMBIA.

del sector industrial y agroindustrial al 52\% del personal como requisito se le exige una experiencia mínima o igual a 12 meses lo que corresponde a un año, al $28 \%$ de los colaboradores su cargo exige un mínimo de 6 meses, al 8\% el máximo de experiencia en el sector que corresponde a 24 meses y al $7 \%$ no se le exige experiencia como requisito para acceder a un cargo determinado (ver tabla 5).

\section{Tabla 5}

Promedio de experiencia que se requiere en el sector industrial y agroindustrial en la ciudad de Villavicencio

\begin{tabular}{lc}
\hline Nivel de educación & Porcentaje \\
\hline Sin experiencia & $7 \%$ \\
6 meses & $28 \%$ \\
12 meses & $52 \%$ \\
18 meses & $5 \%$ \\
24 meses & $8 \%$ \\
\hline
\end{tabular}

Fuente: Elaboración propia.

Dentro de las organizaciones industriales y agroindustriales con una categoría de cargo auxiliar, el $26 \%$ de los colaboradores de dichas empresas se encuentran dentro del cuartil 1 , devengando un salario inferior o igual a \$820.000,00 al año 2017 representa cerca de 1,11 salarios mínimos tenido en cuenta que para dicho año era de $(\$ 737.700,00)$; lo que representa que es lo mínimo y justo que se debe pagar a un colaborador. Dentro de dicho cuartil, existe un amplitud extensa lo que quiere decir que existe una gran variación y desigualdad dentro de la misma categoría, lo que puede influir en que aquellos colaboradores que se encuentran más a la izquierda se encuentren menos motivados ya que a comparación a lo que están más a la derecha estos están ganando menos y por consiguiente en virtud de dicho caso es posible deseen a corto plazo desertar de dicha labor; lo que afecta enormemente las finanzas de las organizaciones ya que representan costos en caso de que exista dicho vacante requiere un nuevo proceso de contratación; el $26 \%$ se encuentra dentro del cuartil 2, lo que representa que devengan un salario superior a \$ 820.000,00 e inferior o igual a \$900.000,00 es amplia la extensión de dicho cuartil, lo cual muestra un pago considerable, ya que está sobre el cuartil 1; mientras que por otro lado, el $47 \%$ de los colaboradores se encuentran dentro del cuartil 3 y devengan un salario mayor a $\$ 900.000,00$ e inferior o igual a $\$ 1.200 .000,00$. En promedio, un colaborador en categoría de cargo auxiliar devenga un salario de \$994.272,55 (ver tabla 6). 
COMPETITIVIDAD SALARIAL DEL SECTOR INDUSTRIAL Y AGROINDUSTRIAL EN LA CIUDAD DE VILLACENCIO, COLOMBIA.

Tabla 6

Rangos de los salarios expresados en cuartiles (2017)

\begin{tabular}{lcccc}
\hline Categoría del cargo & Cuartil 1 & Cuartil 2 & Cuartil 3 & Promedio \\
\hline Auxiliar & $\$ 820.000,00$ & $\$ 900.000,00$ & $\$ 1.200 .000,00$ & $\$ 994.272,55$ \\
Asistente & $\$ 935.000,00$ & $\$ 950.000,00$ & $\$ 1.100 .000,00$ & $\$ 1.040 .000,00$ \\
Supervisor & $\$ 1.725 .000,00$ & $\$ 1.850 .000,00$ & $\$ 1.925 .000,00$ & $\$ 1.800 .000,00$ \\
Profesional & $\$ 2.515 .000,00$ & $\$ 3.000 .000,00$ & $\$ 3.150 .000,00$ & $\$ 2.993 .571,43$ \\
Jefe de primera línea & $\$ 900.000,00$ & $\$ 900.000,00$ & $\$ 1.700 .000,00$ & $\$ 1.433 .333,33$ \\
Administrador & $\$ 900.000,00$ & $\$ 950.000,00$ & $\$ 1.012 .500,00$ & $\$ 962.500,00$ \\
\hline
\end{tabular}

Fuente: Elaboración propia.

El 56\% de los colaboradores que su categoría es de asistente, están dentro del cuartil 1, devengando un salario menor a $\$ 935.000,00$, lo que representa que existe una amplia distancia entre cuartiles y salarios, un factor desmotivante para los colaboradores puesto que mientras están dentro de la misma categoría existe diferencias considerables entre unos y otros, por consiguiente es necesario que la organización haga revaluación de dichos salarios para que la amplia brecha existente sea reducida, por consiguiente los salarios sean más justos y equitativos, así como también colaboradores más comprometidos y productivos, el 1\% se encuentra dentro del cuartil 2, lo que representa que devengan un salario superior a \$935.000 e inferior o igual a $\$ 950.000,00$, lo que representa que es casi nula la brecha existente entre uno y otro cargo dentro de dicha categoría; el 15\% de los colaboradores se encuentran dentro del cuartil 3, devengando un salario mayor a $\$ 950.000,00$ e inferior o igual a $\$ 1.100 .000,00$, mientras que el $28 \%$ son colaboradores que su salario es superior a $\$ 1.100 .000,00$. En promedio, un colaborador en categoría de cargo asistente devenga un salario de $\$ 1.040 .000,00$.

El $66,6 \%$ de los jefe de primera línea que laboran dentro de las organizaciones industriales y agroindustriales se encuentran dentro del cuartil 1 y 2 devengando un salario inferior o igual a \$ 900.000,00 dicho caso se presenta por la poca participación e información acerca de dicho cargo dentro del sector lo que muestra que la brecha existente es casi nula y que los salarios devengados para tal categoría están relevantemente bajos, mientras que el 33,3\% son colaboradores que su salario es superior a $\$ 1.700 .000$ lo que representa que es mayor al cuartil 3. En promedio, un jefe de primera línea devenga un salario de $\$ 1.433 .333,00$ (ver tabla 6 ). 
COMPETITIVIDAD SALARIAL DEL SECTOR INDUSTRIAL Y AGROINDUSTRIAL EN LA CIUDAD DE VILLACENCIO, COLOMBIA.

Los cargos que requieren un nivel de educación profesional son cargos que requieren una mayor experiencia equivalente a 20 meses en promedio; a excepción del empírico, todos los niveles de educación, como son: primaria, bachiller, técnico, tecnólogo y profesional, son directamente proporciones y es clara la existencia de una correlación entre experiencia y nivel de educación, ya que entre mayor nivel de educación mayor será la experiencia expresada en meses que debe tener un colaborador para el desarrollo de sus funciones dentro de la organización.

A excepción de aquellos cuyo nivel de educación es empírica y aquellos que son técnicos que devengan en promedio $\$ 1.126 .000,00$ y $\$ 934.523,00$, respectivamente, tanto quienes tienen primaria, como aquellos que tienen bachiller, tecnólogo o profesional su salario depende directamente de su nivel de educación es decir que existe relación directamente proporcional entre nivel de educación y el salario que dicho colaborador devenga.

En cuanto al promedio de salario que devenga un colaborador en dichos sectores, frecuentemente depende de su nivel de educación, en donde un profesional en promedio devenga \$2.731.111,11 un equivalente a 3,7 salarios mínimos legales vigentes al año 2017 en Colombia (\$737.700,00), en donde como tecnólogo con \$1.202.000,00, técnico con \$ 937467,27 y bachiller con $\$ 937467,27$ resultan un referente bien pago en comparación al salario mínimo equivalentes al 1.6; 1.2 y 1.2, respectivamente, aunque por otro lado muestra que el empírico devenga e incluso mucho más que quien requiere mínimo primaria, bachiller o técnico, con un salario promedio de \$1.126.000.00 que equivalen a 1,5 salarios mínimos legales vigentes.

\section{Condiciones de contratación de personal}

Dentro de las organizaciones de los sectores industriales y agroindustriales, frecuentemente se hace de manera directa, en donde el 92\% del personal es contratado de dicha forma y en donde tan solo el $8 \%$ se hace por medio de terceros, el 66\% del personal es a término indefinido, siendo un factor de incertidumbre que afecta la estabilidad laboral de dicho porcentaje del personal, el $21 \%$ por otro lado, cuenta con un contrato a término fijo; lo que representa estabilidad por el tiempo pactado y lo que motiva a ser más productivo para lograr a fin de contrato una renovación, el 10\% labora bajo labor u obra y tan solo el $3 \%$ de los contratos se hacen bajo términos verbales.

\section{Aspectos salariales}

En promedio, dentro de las organizaciones del sector industrial y agroindustrial, los colaboradores laboran 5,6 días, equivalentes a 44.8 horas a la semana; en términos generales, el sector está 
COMPETITIVIDAD SALARIAL DEL SECTOR INDUSTRIAL Y AGROINDUSTRIAL EN LA CIUDAD DE VILLACENCIO, COLOMBIA.

bien, ya que legalmente los colaboradores deben laborar 48 horas semanales, por otro lado el $69 \%$ de colaboradores laboran sus 6 horas legalmente vigentes y el 26\% menos días a lo establecido por la ley; aunque preocupantemente, el 3\% labora más del promedio y legalmente establecido lo que representa una sobrecarga laboral.

El 99\% de las empresas dentro del sector industrial y agroindustrial, realizan el pago de la nómina a los trabajadores puntualmente de acuerdo a lo establecido en el contrato, ya sea semanal, quincenal o mensual. Por otro lado, el 1\% lo hace dentro de los 5 días después de la fecha acordada. En dicho caso, el 85\% de las organizaciones optan tanto por la transferencia y el cheque como las modalidades más habituales parahacer pago de la nómina laboral; mientras que por otro lado, el efectivo aún sigue siendo una modalidad que no requiere de intervención de terceros, sino simplemente una transacción directa con el colaborador y quien efectúa el pago; modalidad que en comparación a la transferencia y cheque que son efectivas y seguras, expone a ambas partes a ser sujetos de pérdida orobo.

\section{Políticas de incentivos y aumentos salariales que se realizan a los colaboradores}

El $100 \%$ de las organizaciones del sector industrial y agroindustrial han optado el salario emocional como alternativa para motivar a sus colaboradores ampliando practicas propias del salario emocional como salario flexible, actividades para empleados, formación continua, días libres, cumpleaños entre muchas más alternativas que ayudan a hacer un ambiente agradable y un personal saludable y motivado

La inflación produce efectos nocivos sobre los ingresos y los egresos fiscales, reduciéndolos en términos reales; es decir, es el aumento que tienen los precios de una economía. Por ello, la necesidad de que las organizaciones hagan ajuste al salario que devengan sus colaboradores, para tanto estar acorde a la ley, así como también permitir a sus colaboradores acceder a un salario justo que permita satisfacer sus necesidades.

\section{CONCLUSIONES}

Las empresas del sector industrial y agroindustrial que casi siempre aplican el salario emocional, no reconocen el término técnico, pero aun así realizan ciertas actividades de dicho tipo de salario.

El salario que obtienen los colaboradores de las empresas del sector servicio, son de acuerdo a su nivel de escolaridad y su nivel de experiencia, debido a que es importante que tengan el conocimiento y las habilidades requeridas para los cargos que van a ocupar. 
COMPETITIVIDAD SALARIAL DEL SECTOR INDUSTRIAL Y AGROINDUSTRIAL EN LA CIUDAD DE VILLACENCIO, COLOMBIA.

Las empresas aun cuentan con una gran cantidad de pagos en efectivo, debido a que no todas las personas tienen igual manejo de las transacciones tecnológicas, siendo importante poco a poco ir implementando sistemas de pago electrónicos donde se pueda contar con mayor control de los procesos realizados por las empresas. Aunque los beneficios sociales sean un gran recurso para contentar y satisfacer a los empleados, hay que saber gestionarlos y concederlos apropiadamente, sin dejar que los trabajadores se acostumbren a un estilo de vida laboral demasiado tolerante, sobrevalorando su libertad personal.

La compensación al personal permite motivar a los colaboradores de forma que sean eficientes; por tal motivo, se puede ver que en la ciudad de Villavicencio las empresas de servicios manejan días y horas de trabajo de acuerdo a lo estipulados por la ley, debido a que es el medio de pago de sus salarios de manera justa, de tal manera, que el nivel de escolaridad y la experiencia sean las adecuadas para el cargo y se obtener mayor productividad.

\section{REFERENCIAS BIBLIOGRÁFICAS}

Acosta, J. (2015). ¿Adiós al salario fijo? Dinero. Recuperado de http://www.dinero.com/edicionimpresa/negocios/articulo/tendencia-empresas-fijar- salarios-fijos-variables/213004

Adams, S. (2007). Teoría de Stacey Adams. Teoría de la equidad. Recuperado de http://adamssuteoria.blogspot.com.co/2007/10/teoria-de-la-equidad.html

Aparicio, S. (2017). Salario emocional. Recuperado de https://www.rcnradio.com/economia/salario-emocional-mejoraria-la-productividad-las empresas-la-calidad-vida-del-trabajador.

Baquero, M. (2015). Empresas esperan contratar más personal el próximo año. La nación. Recuperado de https://www.nacion.com/economia/negocios/empresas-esperan- contratarmas-personal-el-próximo- ano/VTQJK6KWAZA3FPDHU6O23VWTSE/story/

Barrera, G. (2015). ¿Con qué regularidad se deben hacer evaluaciones de desempeño? Evaluar. Recuperado de http://blogs.evaluar.com/\%C2\%BFcon-que-regularidad-se-deben-hacerevaluaciones-de-desempe\%C3\%B10

Chiavenato, I. (2012). Introducción a la teoría general de la administración (7ª ed.). Recuperado 
COMPETITIVIDAD SALARIAL DEL SECTOR INDUSTRIAL Y AGROINDUSTRIAL EN LA CIUDAD DE VILLACENCIO, COLOMBIA.

de https://es.scribd.com/doc/41375036/Chiavenato-Introduccion-a-La-Teoria-General-deLa-Administracion

Definición Tamaño Empresarial Micro, Pequeña, Mediana o Grande. (mayo, 2012) Mipymes, Gobierno de Colombia. Recuperado de http://www.mipymes.gov.co/ publicaciones/2761/definicion_tamano_empresarial_micro_pequena_mediana_o_grande

Delgado, C. (2004). La compensación total flexible: Conquistar el talento en el siglo XXI (Tesis doctoral, Universitat Abat Oliba Ceu). Recuperada de http://www.tdx.cat /bitstream /handle/10803/9359/Tcdp.pdf?sequence=1

Diez, A. (2014). Siete motivos por los que las empresas deben contar con mujeres en sus plantillas. 20 minutos (marzo, 2014). Recuperado de https://www.20minutos.es/noticia /2076218/0/motivos-empresas/contratar-mujerestrabajadoras/beneficios-equiposdirectivas/

Formación Profesional vs Experiencia Laboral. (2014). Trabajando. Recuperado de http://blog.trabajando.pe/consejos/842-formacion-profesional-vs-experiencia- laboral

Gómez, K. (2017). ¿Qué es el salario emocional y cómo puede mejorar la productividad? Dinero. Recuperado de http://www.dinero.com/empresas/articulo/salario emocional-aumenta-laproductividad/247849

Guihard. (2016). Programas de incentivo laborales. Medellín: Perfil de Coyuntura Económica.

Keynes. (2000). La estructura lógica de la teoría general de keynes. Cuad. Econ,19(33) Recuperado de Scielo: http://www.scielo.org.co/scielo.php?script=sci_arttext\&amp;pid =S0121 47722000000200002

Keynes, J. (2003). Teoría general de la ocupación, el interés y el dinero. Reino Unido: Palgrave Macmillan

Locke, E. (1968). La teoría de todas las metas. Psicología-Online. Recuperado de http://www.psicologia- online.com/pir/teoria-del-establecimiento-de-metas.html

Lombana, J. y Silvia, R. (2008). Marco analítico de la competitividad Fundamentos para el estudio de la competitividad regional. Pensamiento y Gestión, 26. Recuperado de http://www.redalyc.org/articulo.oa?id=64612291002

Los empleados que más ganan. (enero 1993). Dinero. Recuperado de http://www.dinero.com/caratula/edicion- impresa/articulo/los-empleados ganan/20888

Marrugo, M. y Pérez, B. (2012). Análisis de la teoría de las expectativas de Víctor Vroom en el TORRES-FLÓREZ D., DUANA-ÁVILA D., ALMEIDA-ESPINOSA A. 
COMPETITIVIDAD SALARIAL DEL SECTOR INDUSTRIAL Y AGROINDUSTRIAL EN LA CIUDAD DE VILLACENCIO, COLOMBIA.

centro de emprendimiento y desarrollo Pedro Romero "Cemprende". (Tesis de pregrado) Recuperado de http://repositorio.unicartagena.edu.co:8080/jspui/bitstream /11227/527/1/Analisis\%20de\%20la\%20teoria\%20de\%20las\%20expectativas\%20de\%20vi ctor\%20vroom.pdf

Martínez, M. (2005). Ajustes y Desajustes Salariales. Aporrea. Recuperado de https://www.aporrea.org/actualidad/a14017.html

Méndez J. (2014). Plusvalía, salario real y eficiencia del trabajo en el sector manufacturero en Colombia. Apuntes del Cenes. 33(57). Recuperado de http://www.scielo.org.co/scielo.php?script=sci_arttext\&amp;pid=S01230532014000100003 Ministerio del trabajo. (2013). Decreto 2616 de 2013. (febrero, 2018). Recuperado de www.icbf.gov.co: capitulo 1-al-4.pdf

Mujika, I. (1998). Teoría de los neoclásicos. Recuperado de http://eco.unne.edu.ar/ economia/catedras/micro1/Unidad9.pdf

Newstrom y Davis. (2010). Grupo y liderazgo. Recuperado de https://es.scribd.com /doc/133004874/Resumen-Newstrom-y-Davis

Ranking de los trabajos mejor y peor pagados en Colombia durante el 2017. (septiembre, 2017). Dinero. Recuperado de https://www.dinero.com/pais/articulo/ranking-de-trabajos-mejorpagados-en-colombia-segun-acrip/248430

Ricardo, D. (1900). Teoría de la subsistencia. Recuperado de http://www.ehu.eus /Jarriola/Docencia/EcoMarx/salario,\%20precio\%20y\%20gananc ia.pdf

Riobó, A. (2017). ¿Cuál es mejor, el contrato de trabajo celebrado a término fijo, o el convenido a término indefinido? Gerencie.com. Recuperado de https://www.gerencie.com/ cual-esmejor-el-contrato-de-trabajo-celebrado-a-termino-fijo-o-el-convenido-a-terminoindefinido.html

Rivera, G. (2016). Cinco ventajas de contar con líderes de experiencia. Recuperado de https://www.merca20.com/5-ventajas-de-contar-con-lideres-de-experiencia-en-unaempresa/

Rodríguez, F. (2015). Razones para contratar procesos de negocios con terceros. Portafolio. Recuperado de http://www.portafolio.co/negocios/empresas/razones-contratar- procesosnegocio-terceros-39438

Rojas, W. (2018). Recursos humanos y evaluación de desempeño. Impacto humano, Perú. 
COMPETITIVIDAD SALARIAL DEL SECTOR INDUSTRIAL Y AGROINDUSTRIAL EN LA CIUDAD DE VILLACENCIO, COLOMBIA.

Recuperado de http://consultoresimpacto.com/recursos-humanos-y-evaluacion- dedesempeno/

Salario. (noviembre, 2017). Gerencie.com. Recuperado de https://www.gerencie.com/ salario.html

Todaro, R., Godoy, L. y Abramo, L. (2002). Desempeño laboral de hombres y mujeres: que opinan los empresarios. Cadernos Pagu. 17-18(2), 197-236. Recuperado de http://www.scielo.br/pdf/cpa/n1718/n17a08

Zayas, P. (2010). Fundamentos teórico metodológicos de la selección. Recuperado de http://www.eumed.net/ 